\title{
Evaluation of HaloDot: Visualization of Relevance of Off-Screen Objects with over Cluttering Prevention on Mobile Devices
}

\author{
Tiago Gonçalves, Ana Paula Afonso, \\ Maria Beatriz Carmo, and Paulo Pombinho \\ Faculdade de Ciências da Universidade de Lisboa, Portugal \\ \{tgoncalves, ppombinho\}@lasige.di.fc.ul.pt, \\ $\{\mathrm{apa}, \mathrm{bc}\} @ \mathrm{di} . \mathrm{fc} . \mathrm{ul} . \mathrm{pt}$
}

\begin{abstract}
The complexity of presenting and exploring large amounts of graphical data, on mobile devices, increases due to their small screen size. To mitigate this problem several approaches have been proposed to give clues about objects that are located off-screen. In this paper we present a user study comparing the Halo off-screen visualization technique with HaloDot, our approach that aims to improve direction awareness, as well as, relevance of offscreen objects, and to avoid cluttering of Halos. The study shows that searching and pointing relevant Points of Interest $(\mathrm{PoI})$ can be achieved faster than with Halo and that the proposed aggregation method is useful.
\end{abstract}

Keywords: Visualization, Mobile Devices, Off-Screen Objects, Relevance.

\section{Introduction}

Presenting and exploring large amounts of graphical data on small screens are key research topics. To search Points of Interest (PoI) in large maps on mobile devices, panning and zooming can be used to explore surrounding areas that are not visible on screen. However, as these techniques are cognitively complex and frequently disorient the user, different approaches to give visual clues of off-screen objects have already been proposed $[1,2,3,4,5]$.

Another important issue in mobile visualization is the development of mechanisms to show the most relevant information to the user, reducing the amount of information displayed to avoid cluttering. The relevance in a mobile context should capture not only the location of an object, but also other contextual factors, such as, temporal constraints and properties or attributes of an object. Research approaches in this topic aim to establish an appropriate distance function that integrate several contextual factors beyond the location, namely semantic and temporal relevance [6,7].

In this paper we describe and evaluate our approach, HaloDot that enriches the Halo off-screen visualization technique [1], aiming to improve direction awareness of off-screen objects, to give hints about their relevance, and, to prevent cluttering when there are a large number of off-screen objects. 
This paper is organized as follows. Section 2 describes related work about offscreen visualization techniques and relevance visualization. Sections 3 present the HaloDot technique. Section 4 describes the experimental evaluation and reports the results. Finally, section 5 points out our conclusions and future work.

\section{Related Work}

There are several approaches to provide hints to the existence and location of objects that are not visible on the display area. They consist of graphical representations, such as, lines, arcs or arrows, disposed along the borders of the display area to convey information about the distance and direction of off-screen objects. Burigat et al. call them Contextual Cues [8]. Some examples are arrows, Scaled and Stretched arrows [2], City Lights [3], Halo [1], EdgeRadar [4] or Wedge [5]. Halo is one of the most known techniques. It consists in surrounding the off-screen objects with rings, which are just large enough to reach into the border region of the visible area. Based on the visible portion of the ring, users can infer the location and the direction of the object at the center of the ring, taking into account the arc position and arc curvature [1] (Fig.1 left).

The Halo has been compared with other off-screen visualization techniques and is considered as a successful technique for awareness of the presence of off-screen objects $[2,4,5]$. However, these studies have also observed that Halo had lower accuracy in some tasks caused either by the underestimating of the distances or a Halo cluttering problem.

In the initial phase of our work [9], we observed that none of the visualization techniques of off-screen objects conveys their relevance. The concept of relevance and how to represent it has also been subject of various research studies. It is not enough to select the most relevant objects but it is essential that the filtered objects show their relevance values [10]. Reichenbacher has suggested the use of "warm" colors, like red and orange, to represent more relevant spots, while the less relevant ones would be represented with "colder" colors. Such practice is also mentioned in [11], that states that colors can be used to represent various meanings, one of them temperature $($ warm $=$ red, cold = blue). Wolfe J.M [12] also points out the importance of color along with other attributes, such as, motion, orientation and size, to guide users' attention.

We aim to provide visual clues (based on color and transparency attributes) to convey information about the relevance and the distance of off-screen objects, i.e., the distance between its location and the area visible on-screen. Moreover, we want to avoid cluttering. We started our work using the Halo technique [4] and a function that returns a value of the relevance of a PoI belonging to $[0,1]$. The value of the relevance of each PoI, is calculated according with the user preferences and his geographic position [7]. This means that the relevance takes into account the distance between the geographic location of the user and the PoI, which is different from the distance represented by the Halo's arc. 


\section{HaloDot Technique}

We have designed HaloDot that adapts and enhance the Halo interaction technique to satisfy the relevance and direction awareness and prevention of the clutter problem when a large number of off-screen objects are presented. In the next sections we describe our solutions and improvements applied to the Halo technique.

\subsection{HaloDot: Improving the Awareness of Direction}

Halo provides location and the distance of the off-screen object based on the size and shape of the visible portion of the arc. Since the distance is already provided by the arc's size, we decided to enrich the Halo indirect direction representation given by the arc's position. We have drawn the arc of the Halo with a small circle at the point of intersection between the Halo's arc and the intrusion border, i.e., the inner limit of the area where the Halos are visible. This approach combines Halo with the direction provided in City Lights technique. Fig. 1 (right) shows an example of this small adaptation, called HaloDot.

\subsection{HaloDot + Color + Transparency: Awareness of Relevance}

Let us consider the following scenario: a user A standing in the center of the visible area wants to find restaurants with preference to Italian ones. Suppose that the result is the off-screen Italian restaurant 1 and the Japanese restaurant 2. Although, object 1 and 2 are equidistant from the user $\mathrm{A}$, restaurant 1 is more relevant than 2 , because the user prefers Italian restaurants. As shown in Fig. 1 left, the Halo technique does not give awareness of the relevance of off-screen objects.

We have decided to use color to represent the relevance of the various off-screen objects, since color is known as a good attribute to guide people's attention. Using a "warm-cold" analogy [10,11], we have decided to color the most "relevant HaloDots" with red (warm/hot), the less relevant with blue (cold) and the objects with an intermediate relevance with purple (tepid).

Fig.1 (right) shows the use of this approach applied to the above scenario. The most relevant object is represented with the red HaloDot and the less relevant is represented with a blue and more transparent HaloDot.
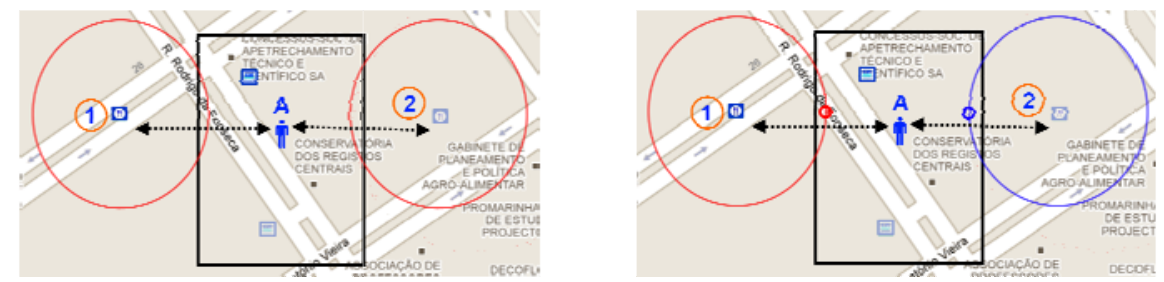

Fig. 1. An example of HaloDot with color. Original Halo (left) and HaloDot (right). 
However, our approach to represent the relevance brings another problem related with the color visibility of the most relevant object. Suppose that the most relevant object 1 (Italian) is further away than the object 2 (Japanese). The original Halo uses transparency to deal with distance [1] and the arc's transparency grows according with the distance of the user to the off-screen object, which means that the arc of the most relevant object will be less visible than the arc of the least relevant (Fig. 1 left).

We decided to apply a minimum transparency level, so even objects that are too far away still have a visible HaloDot. Assuming that the more visible, the more relevant an object is [10], if the transparency level was selected only based on the distance of the off-screen object to the visible area, there would be the risk that, if a relevant (red) object off-screen was further away than a less relevant (blue) one, the second HaloDot's arc would be more visible. This could induce the user to pick the wrong object. To avoid this, the transparency level is also dependent on the object's relevance. An interval of minimum and maximum transparency is set according with the object's relevance. This way, a relevant object will always have a more visible HaloDot than a less relevant one (Fig. 2 right).
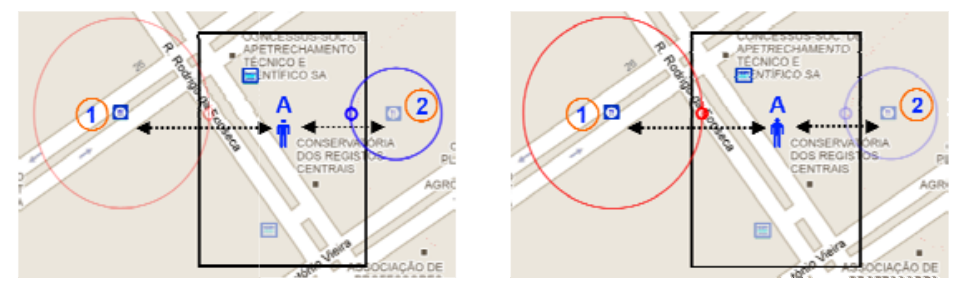

Fig. 2. HaloDot without (left) and with (right) color+transparency to show relevance

\subsection{HaloDot Aggregation + Numbering: Cluttering Prevention}

As mentioned by several authors [1, 2, 5] one of the Halo's limitations is the cluttering problem when a large number of off-screen objects are presented. Although the use of the color and transparency characteristics of HaloDot could reduce clutter by allowing users to visually separate the most relevant, when the number of offscreen objects is very large the visualization could be very difficult (Fig. 3 left).

In resemblance of what is done with on-screen icons cluttering [7], we propose an aggregation approach to mitigate the clutter problem when arcs density is high (Fig. 3 right). We consider the existence of a hypothetical grid, based on geographic coordinates, overlaying the map, which divides the geographical space into cells. The grid has a default size, though it can be changed by the user. Unlike the previous situation, where a HaloDot would be drawn for each off-screen object, a HaloDot will be drawn for each cell with at least one object. This means that, in the worst case, we will have as many HaloDots as cells. The color and transparency shown by a HaloDot corresponds to the most relevant object it represents. 

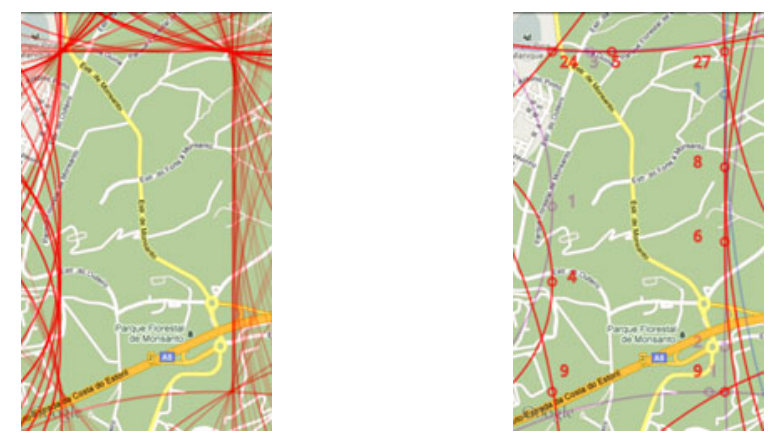

Fig. 3. Halo (left) and HaloDot with aggregation and number clues (right)

To show the number of objects represented by a HaloDot with aggregation we have investigated several approaches. We tried to change the thickness of the arc and/or the point of intersection based on the number of objects represented. However, these demonstrated being very intrusive, even incomprehensible. We have developed another solution that gives textual information, the number of off-screen objects it represents, near the point of intersection with the intrusion border (Fig. 3 right).

To overcome the cluttering problem at the corners, in analogy with EdgeRadar, where the corners represent a larger off-screen area than the borders [4], we have decided to merge all HaloDots on the corners. Although this means that the HaloDots at the corners might represent more points, since they correspond to bigger cell areas and that the aggregations may change by panning the map, we believe that this will improve the technique, since it greatly reduces the overlap and the intrusiveness of the HaloDot, therefore, improving interaction.

Even with this merging, there is the risk that some HaloDots, located in different cells, have centers with a close latitude or longitude, meaning they can overlap the HaloDots and their textual information. To solve this problem we have considered two approaches: to aggregate all HaloDots that are in cells arranged orthogonally to the borders or to aggregate the HaloDots that have their intersection points too close.

Another problem is related with the representation of the center of the aggregated HaloDot. We have explored two options: the center being the midpoint of the objects represented or the most relevant object. While the first may be more intuitive, the second guides the user's attention to the most relevant objects of his/her search, while still not hiding information about the others.

\section{User Study}

We have conducted a user study to evaluate whether the proposed solutions assist the user in searching for off-screen objects according with their relevance. We intend to explore and validate our current solutions for the improvement of the representation of the direction, the representation of the relevance and the aggregation to solve the problem of cluttering, as well as to collect users' preferences about our approaches. To compare the HaloDot improvements with the original Halo, we have done a experiment where tasks were presented to participants through scenarios. 
We also want to understand the best solution when several design alternatives are explored, namely: aggregation of HaloDots based on the orthogonally cells arrangement or based on closeness of intersection points; always display the number of objects represented by the HaloDot or only if there exists more than one off-screen object; representation of the center of the HaloDot as the most relevant object or as the midpoint of the objects represented.

Considering these objectives, our assumptions in the study were the following:

1. Once we have explained the meaning of colors, we expect that users will not have any difficulties identifying the relevance of PoI. We also expect that users find the most relevant PoI faster that using original Halo.

2. The aggregation is a useful method and the users will prefer the aggregation of HaloDots based on cells arranged orthogonally to the borders, since that means, there are less objects being drawn.

3. The users will prefer the center of the HaloDot being the most relevant object, since that object satisfies their information need.

4. The users will prefer to always see the textual information of the HaloDot, because they will find it easier to work with it.

\subsection{Participants and Apparatus}

This study had the participation of sixteen volunteer subjects balanced in gender ( 7 female, 9 male) and background (10 from computer science and 6 from other scientific disciplines and humanities). Their age ranged from 21 to $51 \quad(\mathrm{M}=27$, $\mathrm{SD}=9,2$ ). All users had some familiarity with mapping applications on the Web, such as Google Maps for planning routes or finding PoI. Seven out of 16 users had used mobile maps applications occasionally and one uses it daily for finding PoI and navigating in city environments. Participants had experience with zooming and panning but none were familiar with halos. Three participants stated they had used off-screen techniques in video games, such as arrows and mini-map.

The study was carried out on a touch-screen HTC Desire device, running the Android OS 2.2 featuring a $1 \mathrm{GHz}$ processor and a 3.7 -in touch screen with $480 \times 800$ of resolution. During the study, the map covered all the available screen area of the device, and it was based on the Google Maps application.

\subsection{Tasks}

The study consisted of five tasks that correspond to different scenarios. Before the test the users were informed about the three visualizations techniques in a tourist map application: no contextual clue about off-screen objects; Halo visualization technique and HaloDot. The tasks were performed in the same order (task 1, 2, 3) but the order of the techniques has been counterbalanced as well as the location and relevance configuration of off-screen PoI to reduce sequence and learning effects.

In the first task the participants were asked to find all the PoI ( 3 restaurants) ordering them in increasing distance from the map center (user position). To execute the task the user had to provide their answer by tapping on the corresponding icon of the PoI. The dependable variable was the time to complete the task and a rating of the difficulty in a five point scale. In addition, at the end of the session the users were also 
interviewed to rate their preference for a particular visualization and if they understood the characteristics of HaloDot, namely, its textual representation.

In the second task the participants were asked to find the most relevant PoI (i.e. PoI with the highest relevance value), namely a specific restaurant (e.g. "Good Pizza"). The number of PoI is exactly the same (20) for each visualization condition. However, in the HaloDot visualization there is only one red arc that corresponds to the most relevant PoI. The dependable variable was the time to complete the task and a rating of the difficulty in a five point scale. In addition, at the end of the session the users were also interviewed to rate their preference for a particular visualization and if they understood the characteristics of HaloDot, namely, the color as the representation of the relevance, and if the most relevant HaloDot arc (red) is the one that guides their attention.

In the third task we intend to compare the use of HaloDot with two different conditions in the number of relevant PoI: in the first condition, the number of PoI with higher relevance (red arc) is one in a total of 20; in the second one: the number of PoI with higher relevance is higher than one.

The fourth task intends to test the effectiveness of the proposed aggregation approach. The users were requested to explore the map freely, three times, with 10, 50 and 124 PoI, respectively. The last task intends to compare the different design alternatives of HaloDot, namely, presentation of the center of the aggregated HaloDot; the presence of the number of PoI awareness and the aggregation method preferred. The participants were interviewed to rate their preference for a particular option.

\subsection{Results}

The values of mean completion times for the first task are reported in Fig.4 (left). We have not noticed any relevant result in this task as there is no different effect of the type of visualization configuration (Halo or HaloDot) in the completion time, beyond the difference between the use of an off-screen visualization and no technique at all. At this point few users have noticed the difference between the Halo and the HaloDot, namely in the point of intersection and only one user did not understand the meaning of the number awareness in the HaloDot technique, probably due the existence of few PoI.

For the second task (Fig. 4 middle) a significant main effect was observed for the "type of visualization". A significant difference was found between Halo and HaloDot. This confirms our first hypothesis: users find the most relevant PoI faster that using original Halo. For the third task and based on Fig. 4 (right) we can see that even with more "relevant HaloDots", the users were able to more quickly find the desired PoI. This also supports our first hypothesis: once we explained the meaning of colors, we expected that users would not have any difficulties identifying the relevance of PoI. Comments collected after the execution of these tasks revealed that, although few users $(31 \%)$ have associated the color clue with the relevance of the object, a large number mentioned that the red HaloDots were more visible and provided clues to guide their attention. 

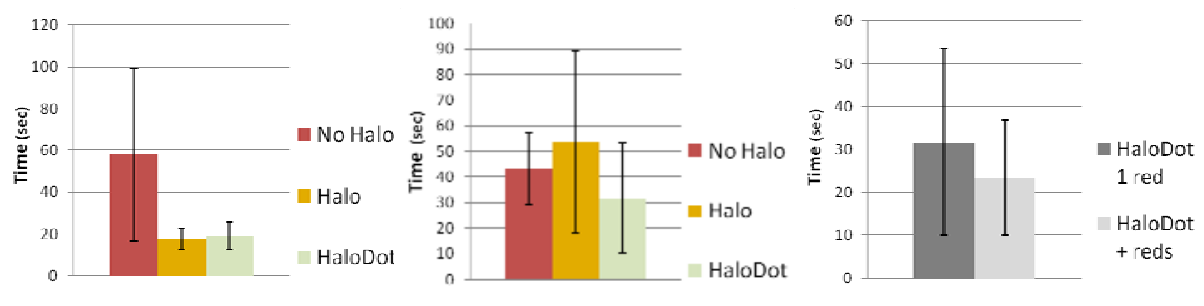

Fig. 4. Mean completion times with standard deviation bars: first task (left); second task (middle); and third task (right)

In the last two tasks, the user expressed their preference between several alternatives of design. In the fourth task, despite the increase of PoI on the map, a large number of users expressed that HaloDot was still usable and that the aggregation was very usefull and $75 \%$ prefered the aggregation based on cells arranged orthogonally to the borders. This confirms our second hypothesis. When asked about their preferences about the various configurations of the HaloDot, the results express a positive evaluation of our third and fourth hypotheses: $75 \%$ prefered to see the HaloDot with a center on the most relevant PoI, 62\% prefered to always see the textual information (number of PoI). The color of the number revealed to be confusing since it was interpreted as the number of the most relevant PoI, instead of being the total number of PoI represented by the aggregate HaloDot, where at least one had a high relevance.

Overall users prefered the HaloDot in comparison with the original Halo, and the main reason pointed was the usage of different colors and the aggregation as they reduce the amount of arcs drawn and consequently improve the perception of the most relevant information. With respect of the utility of the point of intersection as an improvement of direction awarness, users have commented that it was a very helpful feature, however some suggested that other shapes could be used, like arrows.

\section{Conclusions and Future Work}

In this paper we described the HaloDot technique to improve cluttering problems and to give relevance clues about the off-screen objects. An Android tourist mobile application was developed to compare the proposed technique with Halo. The study showed that HaloDot enables users to search faster for relevant PoI than Halo and that aggregation is a useful method. The next step of this work is to perform a more extended and precise comparison with other variations of the off-screen objects representations (e.g. arrows, lines) and relevance hints. This will enable us to understand and how to optimize them to represent relevance of off-screen objects.

\section{References}

1. Baudisch, P., Rosenholtz, R.E.: Halo: a technique for visualizing off-screen objects. In: Proceedings of CHI 2003, pp. 481-488 (2003)

2. Burigat, S., Chittaro, L., Gabrielli, S.: Visualizing location of off-screen objects on mobile devices: A comparative evaluation of three approaches. In: Proceedings of MobileHCI 2006, pp. 239-246 (2006) 
3. Zellweger, P.T., Mackinlay, J.D., Good, L., Stefik, M.J., Baudisch, P.: City Lights: contextual views in minimal space. In: Extended Abstracts CHI 2003, pp. 838-839 (2003)

4. Gustafson, S.G., Irani, P.P.: Comparing visualizations for tracking off-screen moving targets. In: Extended Abstracts of CHI 2007, pp. 2399-2404 (2007)

5. Gustafson, S., Baudisch, P., Gutwin, C., Irani, P.: Wedge: clutter-free visualization of offscreen locations. In: Proceeding of CHI 2008, pp. 787-796 (2008)

6. Swienty, O., Reichenbacher, T., Reppermund, S., Zihl, J.: The role of relevance and cognition in attention-guiding geovisualisation. The Cartographic Journal 45(3), 227-238 (2008)

7. Pombinho, P., Carmo, M.B., Afonso, A.P.: Evaluation of Overcluttering Prevention Techniques for Mobile Devices. In: Proceedings IV 2009, pp. 127-134 (2009)

8. Burigat, S., Chittaro, L.: Visualizing references to off-screen content on mobile devices: A comparasion of Arrows, Wedge and Overview + Detail. Interacting with Computers 23, 156-166 (2011)

9. Gonçalves, T., Afonso, A.P., Carmo, M.B., Pombinho, P.: HaloDot: Visualization of the Relevance of Off-Screen Objects. In: SIACG 2011, pp. 117-120 (2011)

10. Reichenbacher, T.: The concept of relevance in mobile maps. Location Based Services and TeleCarthography, 231-246 (2007)

11. Silva, S., Santos, B.S., Madeira, J.: Using Color in Visualization: A Survey. Computer \& Graphics 35(2), 320-333 (2011)

12. Wolfe, J.M., Horowitz, T.S.: What attributes guide the deployment of visual attention and how do they do it? Nature reviews. Neuroscience 5, 1-7 (2004) 